\title{
Local synteny and codon usage contribute to asymmetric sequence divergence of Saccharomyces cerevisiae gene duplicates
}

Lijing Bu, Ulfar Bergthorsson and Vaishali Katju*

\begin{abstract}
Background: Duplicated genes frequently experience asymmetric rates of sequence evolution. Relaxed selective constraints and positive selection have both been invoked to explain the observation that one paralog within a gene-duplicate pair exhibits an accelerated rate of sequence evolution. In the majority of studies where asymmetric divergence has been established, there is no indication as to which gene copy, ancestral or derived, is evolving more rapidly. In this study we investigated the effect of local synteny (gene-neighborhood conservation) and codon usage on the sequence evolution of gene duplicates in the $S$. cerevisiae genome. We further distinguish the gene duplicates into those that originated from a whole-genome duplication (WGD) event (ohnologs) versus smallscale duplications (SSD) to determine if there exist any differences in their patterns of sequence evolution.

Results: For SSD pairs, the derived copy evolves faster than the ancestral copy. However, there is no relationship between rate asymmetry and synteny conservation (ancestral-like versus derived-like) in ohnologs. mRNA abundance and optimal codon usage as measured by the CAI is lower in the derived SSD copies relative to ancestral paralogs. Moreover, in the case of ohnologs, the faster-evolving copy has lower CAI and lowered expression.

Conclusions: Together, these results suggest that relaxation of selection for codon usage and gene expression contribute to rate asymmetry in the evolution of duplicated genes and that in SSD pairs, the relaxation of selection stems from the loss of ancestral regulatory information in the derived copy.
\end{abstract}

\section{Background}

The appearance of novel biochemical traits contributing to phenotypic diversity is inextricably linked with the constant input of new genetic fodder via gene and genome duplication. However, a mere duplication of an ancestral locus far from guarantees the origin of a novel gene product and the majority of gene duplicates end up being silenced following a brief evolutionary existence $[1,2]$. For those paralogs that emerge unscathed by deleterious mutations, the first clues as to how paralogs are able to forge an independent evolutionary trajectory may be provided by studying their patterns of expression divergence and relative rates of molecular evolution.

\footnotetext{
* Correspondence: vkatju@unm.edu

Department of Biology, University of New Mexico, Albuquerque, NM 87131,
} USA

(C) 2011 Bu et al; licensee BioMed Central Ltd. This is an Open Access article distributed under the terms of the Creative Commons Attribution License (http://creativecommons.org/licenses/by/2.0), which permits unrestricted use, distribution, and reproduction in any medium, provided the original work is properly cited.
Early studies of DNA sequence divergence between paralogs suggested there was little or no difference between duplicate gene-copies in their rates of evolution [3-7]. These results were used to argue against the hypothesis proposed by Ohno that following gene duplication, one copy is under relaxed selection and begins to accumulate previously 'forbidden' mutations [2]. However, these analyses may have had limited power to detect differences in evolutionary rates, or rate asymmetry, because they analyzed old duplicates, while an increase in the evolutionary rate is easiest to detect in young gene duplicates [8]. Subsequent studies have demonstrated relatively large rate asymmetry between duplicate genes [9-13]. For instance, 20\%-30\% of paralocant differences in evolutionary rate [11] and one or of the cases [12]. gous gene in Saccharomyces cerevisiae displayed signifiboth paralog(s) exhibited accelerated evolution in 17\%
C Biomed Central 
The phrase "gene duplication" appears to imply that all functionally relevant features of an ancestral gene are duplicated and therefore the two resulting gene copies ought to be functionally equivalent. In fact, there may be numerous differences between the two "copies". The derived copy often does not retain the full regulatory element repertoire of the ancestral copy or has some structural or genomic location differences relative to the ancestral gene [8,14-16]. These differences suggest that the derived copy might be expected to evolve under divergent constraints relative to the progenitor gene, either due to relaxation of natural selection or due to selection for novel attributes. In the majority of studies where asymmetric divergence has been established, there is no indication as to which gene copy, ancestral or derived, is evolving more rapidly. 'Derived' and 'ancestral' in the context of this study refer to the location of the paralogs in the genome rather than function. Recently, a study of gene duplicates in the mouse genome found that relocated gene copies following duplication, and in particular retrotransposed copies, evolved faster than paralogs in their ancestral location [16]. Similarly, a study in four mammalian genomes found that genes that came to reside in a different location following gene duplication were more likely to display evidence of adaptive evolution relative to gene copies that did not relocate [17].

In the case of a new gene-copy originating from a small-scale duplication (SSD) event and relocating some genomic distance from the ancestral copy, the identity of the ancestral and derived copies can be established by conservation of synteny flanking the paralogs or chromosomal location in comparison to a single-copy ortholog in an outgroup genome $[15,16]$. Distinguishing the ancestral from the derived copy becomes problematic in the case of whole-genome duplication (WGD henceforth). For example, in the instance of a genome resulting from allopolyploidy where duplicate gene-copies result from hybridization rather than gene duplication, naming ancestral and derived genes has no biological relevance.

Here we examine paralogs with low synonymous divergence in the S. cerevisiae genome to determine if it is the derived copy that evolves faster than the ancestral copy following gene duplication. Most duplicates in yeast originated from a WGD event $[12,18]$ and for reasons mentioned in the preceding paragraph, it is inappropriate to assign ancestral and derived status to gene copies in the same manner as duplicates arising from SSD events. Gene duplicates that were previously identified as resulting from the WGD event are henceforth referred to as 'ohnologs' and were analysed separately from those resulting from SSD events to test if these two pools of duplicated genes behaved differently with respect to their rates of molecular evolution.

\section{Results}

\section{Greater conservation of synteny in ohnologs}

We initially commenced the analysis with 43 pairs of ohnologs and 15 SSD-derived gene duplicate pairs. These only included gene pairs that could be unambiguously assigned a single ortholog in an outgroup genome and the identification of local synteny conservation. Despite massive gene loss and genomic rearrangements in the evolutionary period subsequent to the WGD event, ohnologs have more extensive tracts of synteny relative to SSD-originated gene duplicates (Table 1). For instance, the average total upstream and downstream number of syntenic genes in the flanking regions for ohnologs versus SSD pairs is 19.87 and 4.67, respectively. Additionally, Wilcoxon signed-ranks tests revealed no significant difference in the extent of syntenic tracts in the upstream and downstream flanking regions within each population of yeast paralogs (ohnologs and SSD pairs).

\section{Rate of molecular evolution of ohnologs is decoupled from synteny conservation}

Nine and zero of 43 ohnolog pairs displayed significant asymmetry based on Tajima's Relative Rate test (uncorrected for multiple comparisons) using DNA (Additional File 1, Table S1) and amino acid sequences (Additional File 2, Table S2), respectively. Of these nine pairs of ohnologs, the faster evolving copy was associated with less synteny conservation in seven instances. This would indicate that the rate of evolution for paralogs formed via polyploidization might be influenced by the degree of preserved synteny. However, a nonparametric rank correlation test testing for association between synteny (sum of upstream and downstream continuous synteny) and the number of unique nucleotide sites was nonsignificant (Kendall's tau $=0.0132 ; p=0.91$ ). Likewise, we found no significant association between synteny preservation and the number of unique sites at the amino acid level (Kendall's tau $=0.0086 ; p=0.94$ ).

Table 1 Averaged measures of synteny preservation for $\mathbf{4 3}$ pairs of ohnologs versus 15 SSD pairs in the S. cerevisiae genome

\begin{tabular}{lccc}
\hline Synteny Measure & Ohnologs & $\begin{array}{c}\text { SSD } \\
\text { pairs }\end{array}$ & p-value \\
\hline Upstream continuous & 1.41 & 0.47 & 0.0002 \\
Downstream continuous & 1.50 & 0.20 & $<0.0001$ \\
Upstream continuous + Downstream & 2.91 & 0.67 & \\
Continuous & & & \\
Upstream total & 10.08 & 3.00 & $<0.0001$ \\
Downstream total & 9.79 & 1.67 & $<0.0001$ \\
Upstream total + Downstream total & 19.87 & 4.67 & \\
\hline
\end{tabular}

For all measures of synteny (upstream continuous, downstream continuous, upstream total, and downstream total), the extent of synteny preservation is significantly greater in ohnologs relative to SSD pairs based on Wilcoxon tests. 
Derived gene copies originating from SSD events exhibit accelerated rates of molecular evolution

Seven of 15 SSD pairs showed significant asymmetry using a Tajima's Relative Rate test at the nucleotide and amino acid level, respectively (Additional File 3, Table S3 and Additional File 4, Table S4). Six of these seven SSD pairs exhibited rate asymmetry both at the nucleotide and amino acid level. In all seven instances of significant rate asymmetry between paralogs at the nucleotide level, the derived copy exhibited accelerated rates of molecular evolution. In six of the seven instances of significant rate asymmetry at the amino acid level, the derived copy was the faster-evolving paralog. A Wilcoxon signed-ranks test of all 15 SSD pairs showed that collectively, the derived copies tend to possess a greater number of unique sites, suggesting accelerated molecular evolution at the nucleotide level $(T=-25.0 ; p=0.024)$ as well as the amino acid level $(T=-21.0 ; p=0.029)$.

\section{CAI Results}

Codon adaptation index (CAI) is a measure of optimal codon usage and it is positively correlated with levels of gene expression [19]. Following gene or genome duplication, there may be a period of relaxed selection resulting in lower CAI. If relaxation of selection does not apply equally to both paralogs, we may observe greater reduction in the use of optimal codons and CAI in one of the paralogs. We tested for the degree of association between the difference in CAI values between the two paralogs and the degree of rate asymmetry at the nucleotide level (difference in unique sites between the two paralogs generated from the Tajima's Relative Rate test) for both pools of gene duplicates in the $S$. cerevisiae genome. For SSD pairs, the derived paralogs have a significantly lower CAI than the ancestral paralogs (Wilcoxon signed-ranks test: $T=39.5 ; p=0.011$ ). However, we did not find a significant association between nucleotide rate asymmetry and change in CAI (Kendall's tau $=0.226 ; p=0.25$ ) (Figure 1). That is, faster-evolving paralogs did not have lower CAI values than slowly-evolving paralogs for SSD pairs. In contrast, we find a strong negative correlation between rate asymmetry and a difference in CAI values among ohnologs (Kendall's tau $=-0.453 ; p<0.0001$ ) (Figure 2). Here, the faster-evolving paralogs resulting from the whole genome duplication event also have lower optimal codon preference.

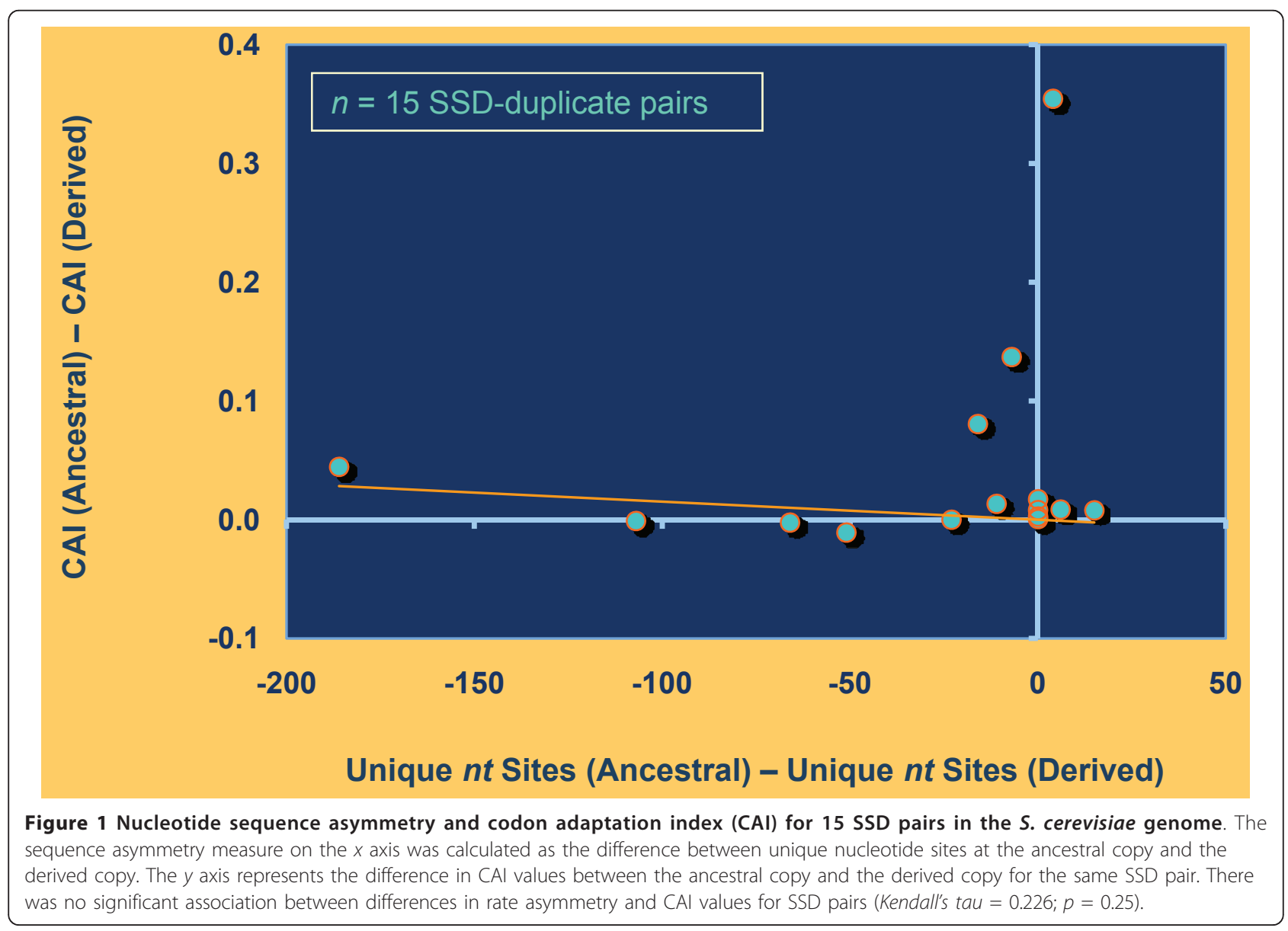




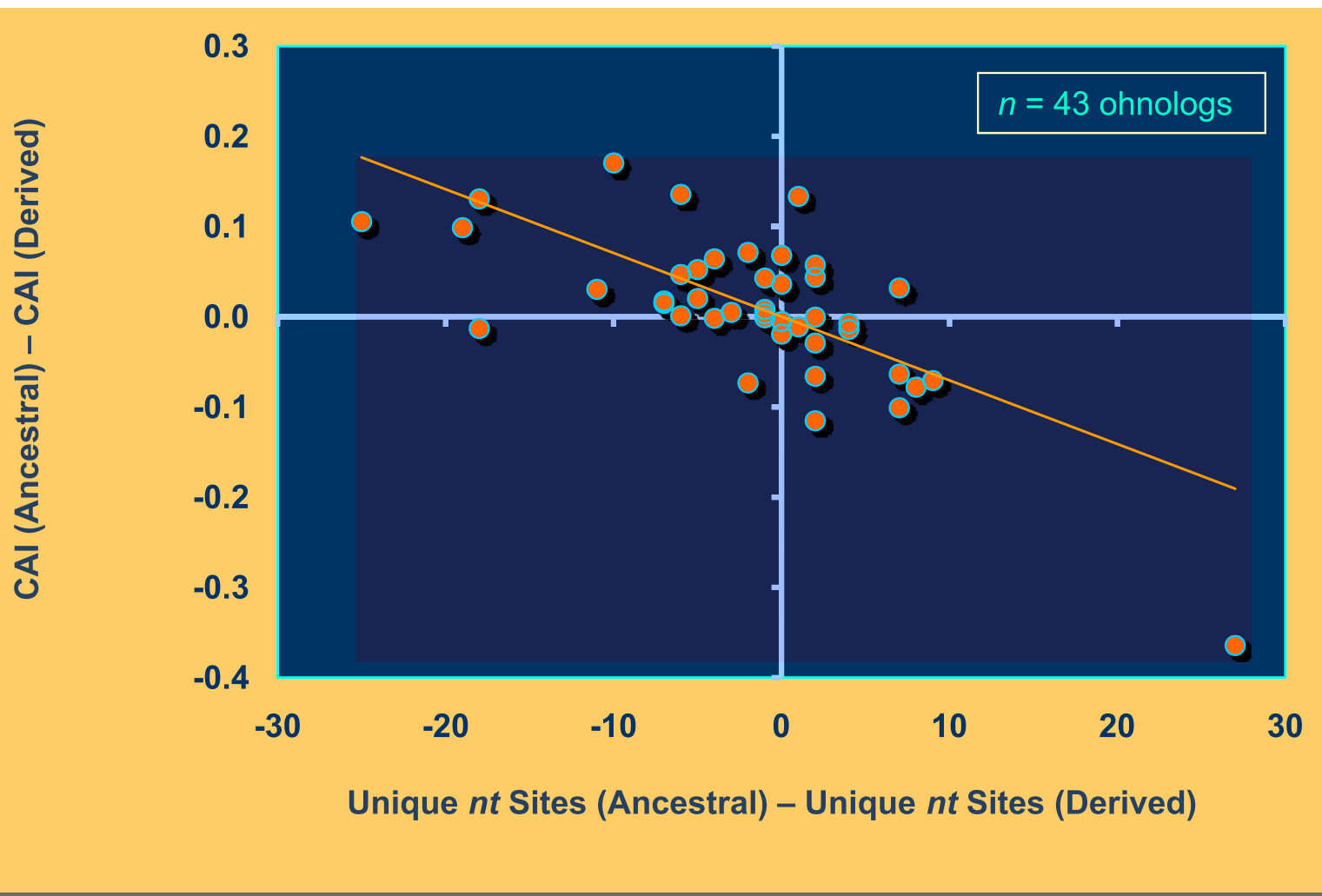

Figure 2 Negative relationship between nucleotide sequence asymmetry and codon adaptation index (CAl) for 43 pairs of ohnologs in the $\mathbf{S}$. cerevisiae genome. The sequence asymmetry measure on the $x$ axis was calculated as the difference between unique nucleotide sites at the ancestral-like copy and the derived-like copy within an ohnolog pair. The $y$ axis represents the difference in CAI values between the ancestral-like copy and the derived-like copy for the same ohnolog pair. There was a significant negative correlation between differences in rate asymmetry and CIA values for ohnologs (Kendall's tau $=-0.453 ; p<0.0001$ )

Ohnologs and SSD duplicate pairs also differ with respect to their CAI values. The median CAI value for ohnologs and SSD pairs are 0.70 and 0.11 , respectively. Indeed, CAI values averaged across both paralogs were determined to be significantly greater for ohnologs relative to SSD pairs (Wilcoxon two-sample test: $\mathrm{Z}=-4.723 ; p<0.0001$ ).

\section{Faster-evolving paralogs have lower mRNA abundance}

The preceding CAI results suggest that relaxed selective constraints due to reduced expression of the derived paralog may contribute significantly to rate asymmetry between ancestral and derived paralogs. We find that ancestral paralogs are expressed at significantly higher levels (greater mRNA abundance) than derived paralogs for SSD pairs (Wilcoxon signed-ranks test: $T=37.5 ; p<$ 0.017). In contrast, ancestral-like ohnologs with greater syntenic preservation do not differ significantly in their expression levels compared to derived-like ohnologs with lower syntenic preservation (Wilcoxon signed-ranks test: $T=52 ; p=0.54)$.

We additionally tested if there is a relationship between transcription levels of paralogs and their degree of rate asymmetry at the nucleotide level. Figure 3 shows a significant correlation between the ratio of paralog-specific RNA and the ratio of unique sites in derived and ancestral copies of SSD pairs ( $r=0.87$, Kendall's tau $=0.74, p<0.0002$ ). Likewise, we find a significant association between the ratio of paralog-specific RNA and the ratio of unique sites in derived and ancestral copies for ohnologs $(r=0.38$, Kendall's tau $=0.225$, $p=0.0343)$.

\section{Discussion}

Duplicated genes frequently experience an initial increase in their rate of evolution and nonsynonymous substitutions relative to synonymous substitutions. Moreover, recent analyses of young gene duplicates in several eukaryotic genomes indicate that paralogs exhibit asymmetric rates of sequence divergence in the evolutionary period soon after duplication [11,16,20-24]. Together, these observations indicate that initial relaxation of selection, or adaptive evolution, after duplication is limited to one of the paralogs, and that the slowerevolving paralog is more constrained by its ancestral 


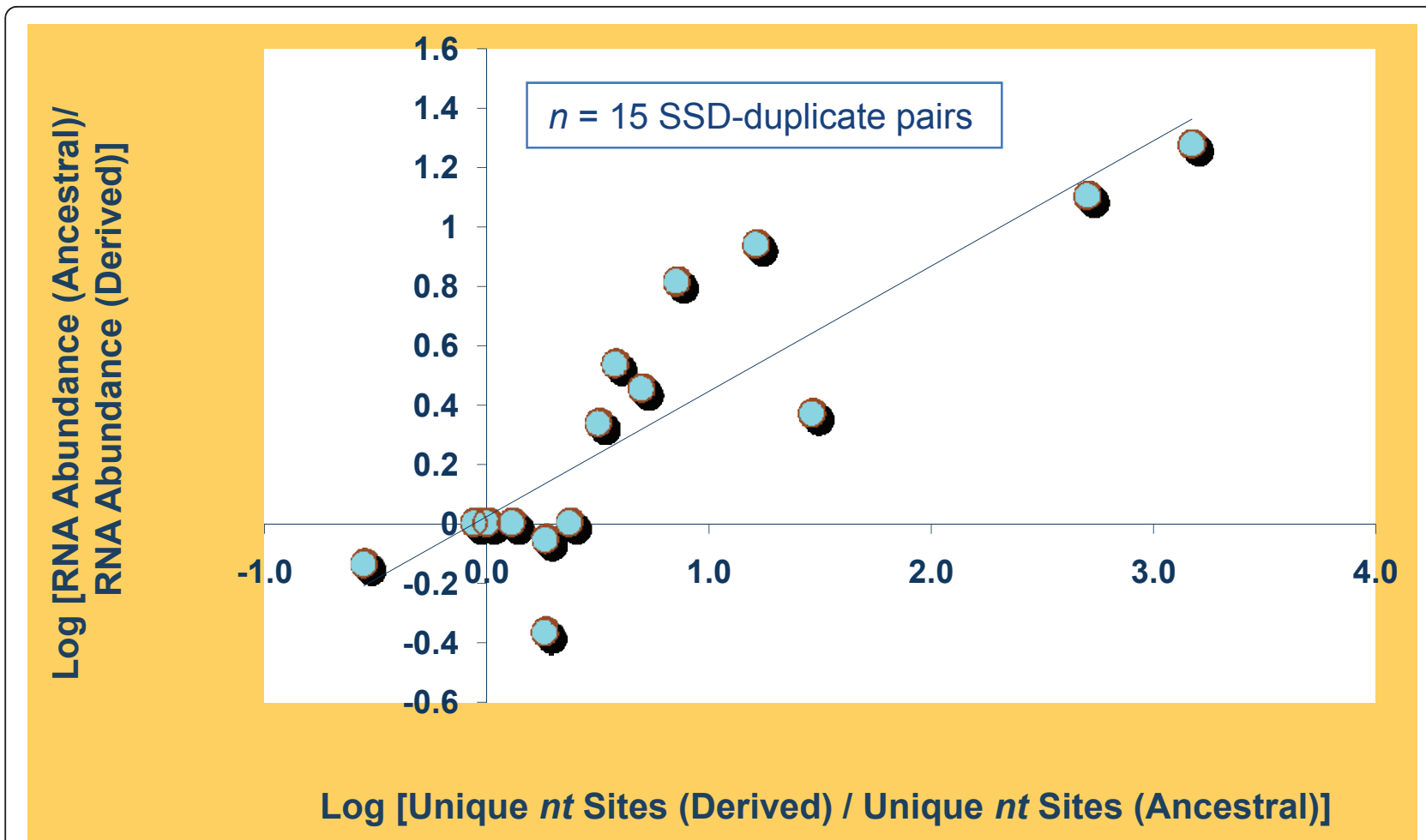

Figure 3 Nucleotide sequence asymmetry and mRNA abundance for 15 SSD pairs in the S. cerevisiae genome. The sequence asymmetry at the nucleotide level is expressed as the $\log _{10}$ (unique sites in the derived paralog/unique sites in the ancestral paralog) and relative RNA abundance is expressed as the $\log _{10}$ (RNA count for ancestral paralog/RNA count for derived paralog). There is a significant correlation between divergence between paralogs at the sequence level and divergence in their expression profiles (as represented by mRNA abundance) (Kendall's $\tan =0.74 ; p<0.0002$

function $[11,22]$. The majority of past studies did not distinguish between the ancestral and derived copies within a gene-duplicate pair, which in turn has precluded an unambiguous assessment of which copy is under stringent versus relaxed selective constraints.

There is some evidence that derived paralogs evolve faster than their counterparts residing at ancestral locations. In their study of evolutionarily young rodent gene duplicates, Cusack and Wolfe [16] assigned ancestral versus derived states to paralogs and demonstrated that genomic relocation of one paralog by retrotransposition engenders rate asymmetry in the sequence evolution of paralogs, commonly manifested as an accelerated rate of sequence evolution in the relocated paralog. Likewise, in bacterial genomes, the majority of paralogs that appear to have moved away from their ancestral gene neighborhood evolved faster than static paralogs [25]. Furthermore, a study of gene duplicates in four mammalian genomes determined that signatures of positive selection were more frequent in the derived copies than genes at their ancestral locations [17].

In this study, we analysed the rate of evolution in yeast paralogs for which an ancestral versus derived status could be assigned by analyzing synteny as manifested in gene-neighborhood conservation. There was significantly greater gene-neighborhood conservation in ohnologs relative to SSD pairs. Although ohnologs originated from an ancient polyploidization event and rampant genome-wide deletions have since restored functional normal ploidy in these Saccharomyces species $[26,27]$, it is noteworthy that this extensive gene-neighborhood conservation has persisted. There is no difference in the extent of gene-neighborhood conservation in the upstream and downstream regions of the paralogs for both populations of duplicates (ohnologs and SSD), suggesting, on average, equal rates of preservation/loss of upstream and downstream neighboring genes.

The majority of gene duplicates with low sequence divergence in S. cerevisiae stem from an ancient WGD event rather than segmental duplications. Subsequent to the WGD event, there has been extensive loss of genetic material with an estimated $10 \%$ of the original ohnologs remaining [12]. Deletions of genetic material within a WGD-derived homology block have the potential to remove or rearrange regulatory sequences for the remaining genes in the block. Therefore, the DNA sequence of a paralog associated with more extensive gene-neighborhood conservation (i.e. local synteny) 
might be under stronger purifying selection than a paralog residing in regions that have endured more gene loss and rearrangements. While it is problematic to assign ancestral versus derived states to gene duplicates originating from WGD events, we reasoned that a paralog within an ohnolog pair could be characterized as being ancestral-like or derived-like based on the extent of gene-neighborhood conservation it shared with a single-copy ortholog in an outgroup genome. We then sought to test the hypothesis that ancestral-like genecopies within ohnolog pairs are more likely to maintain ancestral gene function and therefore exhibit lower rates of sequence evolution. In contrast, gene-copies displaying a reduction in the extent of local synteny relative to the ortholog may be predisposed to accelerated rates of sequence evolution and the resultant fates of neofunctionalization or nonfunctionalization. However, we find no evidence of an association between rate asymmetry in ohnologs and local gene-neighborhood conservation. In other words, for ohnologs, a decline in local geneneighborhood conservation (derived-like) does not engender accelerated rates of sequence evolution either at the nucleotide or amino acid level. This is in contrast to a study of vertebrate genomes that found a significant correlation between synteny preservation and sequence conservation [28]. We speculate that the greater number of regulatory sites in vertebrate genomes might engender greater sensitivity to syntenic changes relative to yeast. However, ohnologs in yeast do exhibit a strong significant relationship between rate asymmetry and CAI such that the faster-evolving paralogs have lower CAI. The rate asymmetry in ohnologs also seems to be to some degree caused by relaxation of selection for codon usage in one copy.

Among the SSD pairs in our sample, it is the derived copy that evolves faster on average, both at the nucleotide and the amino acid level. This lends credence to Ohno's original hypothesis that duplication enables redundancy, enabling one copy to explore new evolutionary space by accumulating mutations [2]. It is likely that segmental duplications frequently do not capture the full repertoire of regulatory sequences [8] associated with the ancestral genes and/or result in the insertion of the derived copy into a region of the genome with different chromatin structure and potentially under the influence of different regulatory elements. Under these conditions, mutations that interfere with the ancestral gene's original function would still be selected against, whereas the derived copy could be under relaxed or positive selection. For SSD pairs, the rate asymmetry at the nucleotide level is likely due to a regime of relaxed selective constraints as there is a significant reduction in the CAI of the derived paralogs within SSD pairs. The CAI compares the codon usage of a gene to codon usage in highly expressed genes; hence, the reduction in the CAI values of derived paralogs suggests that selection for optimal codon usage has been relaxed in the derived copy. Puzzlingly, we failed to detect any correlation between nucleotide sequence asymmetry of SSD paralogs and changes in their CAI values. This may stem from limited power given the small sample size of available SSD duplicates in the yeast genome.

If the rate asymmetry in paralogs is largely a consequence of relaxation of selection in the derived paralog, it should also be manifested as different levels of expression among the two copies. Previous work has shown that the evolutionary rate in yeast is strongly influenced by gene expression $[29,30]$. In both the yeast ohnologs and SSD pairs studied here, mRNA abundance is correlated with the rate of evolution. Moreover, within SSD pairs, it is the derived paralogs that have lowered mRNA abundance relative to the ancestral loci. Both the CAI and mRNA abundance suggest that selective constraints on gene expression is a significant driver of evolutionary rate asymmetry in paralogs.

\section{Conclusions}

Following gene duplication, there is a general increase in the rate of evolution, and this increase is frequently asymmetric in that one paralog evolves at an accelerated pace. Asymmetry in the rate of molecular evolution after duplication has been variously associated with the evolution of novel functions, change in the number of interactions, and relaxation of selection. Here we address the related question if certain factors predispose one paralog to evolve faster. For instance, segmental duplications may translocate the derived copy to a different regulatory environment where it may evolve under different or reduced constraints [8]. Despite a limited sample of gene-duplicate pairs originating from recent small-scale duplications in S. cerevisiae, we find that the derived copy tends to evolve faster and is under reduced selection for codon usage. Accelerated rates in ohnologs are also associated with reduced selection for codon usage. Moreover, the rate of evolution is negatively correlated with mRNA abundance for ohnologs as well as SSD pairs. This adds to the evidence from mammals [17] that genes are not born equal and that the duplication process predisposes the derived copy to an evolutionary trajectory of initially reduced selective constraints and one that is perhaps more conducive to the evolution of new functions.

\section{Methods}

Identification of Gene Duplicates in S. cerevisiae with Low Synonymous Divergence

We initially selected gene families in the S. cerevisiae genome identified in a preceding study [31] that 
comprised only two members and synonymous divergence $\left(K_{S}\right) \leq 0.35$. This set had been extracted via the Genome History program [32] using the following parameters: (i) minimum translated ORF length of 100 aa, (ii) minimum number of aligned residues to accept pair being 100 aa, and (iii) using the BLAST matrix BLOSUM62 and acceptance of all BLAST hits with e $\leq 1 \mathrm{e}$ 07. The majority of gene duplicates within this initial sample were identified as 'ohnologs' [33] or duplicates originating from a WGD event [12,34-37]. To further increase representation of gene duplicate pairs originating from small-scale duplication (SSD) events, we raised the $\mathrm{K}_{S}$ cut-off to 1.0 for two-member families and additionally included three-member gene families with $\mathrm{K}_{S}$ cut-off equal to 0.35 . Ohnologs and SSD pairs in S. cerevisiae were distinguished by consulting Byrne and Wolfe's reconciled ohnolog list from recent comparative genomics studies [36]. The initial dataset after this first set of filtering procedures comprised 47 ohnologs and 31 SSD pairs.

\section{Determination of the extent of synteny preservation with outgroup genomes}

Synteny blocks (regions of conserved gene order) were retrieved on the YGOB database http://wolfe.gen.tcd.ie/ ygob/). For ohnologs, the single-copy ortholog within the reconstructed ancestor chromosome that is hypothesized to exist immediately before the occurrence of the WGD event 100-200 mya [37] was used as a reference outgroup. For SSD-originating paralogs, the sequence of the most recent ancestor of the paralogs was inferred based on related genes in seven post-WGD yeast species (Saccharomyces paradoxus, S. mikatae, S. kudriavzevii, S. bayanus, S. castellii, Candida glabrata, and Kluyveromyces polyspora) using the codeml program of PAML by the setting the RateAncestor $=1$ [38-40]. Tajima's Relative Rate test was then performed using DNA and protein sequences in triplets containing the two focal $S$. cerevisiae paralogs and their inferred ancestral sequence. In addition, duplications involving more than one gene locus, also referred to as 'linked sets' [31] were treated as a single duplication.

We used two measures to quantify the extent of geneneighborhood conservation of each S. cerevisiae paralog in its upstream and downstream flanking regions. The first measure tallied the number of continuously shared genes with the outgroup genome in both the upstream and downstream directions. The second measure tallied the total number of genes shared with the outgroup genome within a block comprising 20 loci in both the upstream and downstream flanking regions. After excluding duplicate pairs with neither synteny nor outgroup information, the sample size of our study comprised 43 and 15 pairs of ohnologs and SSD-originated duplicates, respectively (Additional Files 1-4, Tables S1-S4).
Determining the degree of asymmetry among paralogs Tajima's Relative Rate test [41], as implemented in MEGA version 4.0 [42] was used to determine if one of the paralogs was evolving faster. For SSD pairs, the designated outgroup sequence was a single-copy ortho$\log$ in an outgroup genome closely-related to S. cerevisiae. In the event that multiple outgroup species possessed a single-copy ortholog corresponding to $S$. cerevisae's paralogs, we selected as outgroup the ortho$\log$ in the most closely-related outgroup genome. With respect to three-member gene families, the Tajima's test was only performed for the two most closely-related gene copies. For ohnologs, the outgroup was the phylogenetically closest species that contained a single-copy ortholog to the S. cerevisiae duplicate pair and diverged from the Saccharomyces sensu stricto group prior to the WGD event.

Genome and protein sequences of 11 fully sequenced yeast species were downloaded from the YGOB http:// wolfe.gen.tcd.ie/ygob/ and KEGG http://www.genome.jp/ kegg/catalog/org_list.html databases. Outgroup identification was performed using DNA and protein sequences of the paralogs as queries in BLASTN and BLASTP searches against the genomic and protein sequences of the 11 yeast species. The BLAST outputs were filtered and organized using a Perl script. Gene duplicate pairs and their associated outgroup sequences were first aligned with ClustalW 2.0 and then manually checked and improved, when necessary, before the analysis.

The Wilcoxon signed-rank test was used to test if, collectively speaking, the ancestral and derived copies of a gene duplicate pair are evolving at the same rate. Since the ohnolog copies could not be classified as ancestral or derived, this tests if the rate of evolution is associated with the conservation of flanking synteny. Five pairs of ohnologs with equal number of unique sites were excluded from the Wilcoxon signed-rank test to yield a final sample of 38 ohnolog pairs. For SSD pairs, the paralog with the greater upstream synteny compared to the outgroup is taken to be the ancestral copy. In the event that both paralogs have equal continuous synteny, the total synteny gene number within 20 gene loci was further included as a measure of synteny conservation. If the information above was insufficient for distinguishing the ancestral and the derived copies, the total synteny within 20 upstream and downstream gene loci was utilized.

\section{Relationship between codon usage, mRNA abundance and rate asymmetry}

The Codon Adaptation Index (CAI) was calculated using the JCat tool http://www.jcat.de[19,43]. The JCat tool uses the method of Carbone and colleagues [44] to select a set of reference genes with optimal codon 
usage. In order to determine if differences in the rates of evolution are related to changes in optimal codon usage, we tested for correlation between the difference in number of unique sites (number of unique sites at the ancestral locus - number of unique sites at the derived locus) and the difference in CAI between paralogs (CAI of ancestral locus - CAI of derived locus).

An association between CAI and rate asymmetry between paralogs would suggest that gene expression is imposing differential constraints on the paralogs. As a proxy for gene expression, we obtained mRNA abundance data for all the paralogs in this study from a dataset consisting of transcript counts using single-molecule sequencing [45]. This data was used to test for an association between mRNA abundance and nucleotide rate asymmetry for both SSD pairs (Figure 3) and ohnologs.

\section{Additional material}

Additional file 1: Table S1. Tajima's Relative Rate Test for Ohnolog DNA sequences.

Additional file 2: Table S2. Tajima's Relative Rate Test for Ohnolog amino acid sequences.

Additional file 3: Table S3. Tajima's Relative Rate Test for DNA sequences of SSD pairs using a maximum-likelihood generated ancestral sequence as outgroup.

Additional file 4: Table S4. Tajima's Relative Rate Test for amino acid sequences of SSD pairs using a maximum-likelihood generated ancestral sequence as outgroup.

\section{Acknowledgements}

The authors gratefully acknowledge the comments of two anonymous reviewers whose comments helped improve the final manuscript. VK was supported by the National Science Foundation (NSF) Fellowship in Biological Informatics (DBI 0532735). UB and VK were supported by National Science Foundation (NSF) grant DEB-0952342.

\section{Authors' contributions}

LB was responsible for the data collection. VK and UB conceived the study. All authors conducted statistical analyses and participated in the preparation of the manuscript. All authors read and approved the final manuscript.

Received: 6 June 2011 Accepted: 28 September 2011

Published: 28 September 2011

\section{References}

1. Haldane JBS: The part played by recurrent mutation in evolution. Am Nat 1933, 67:679-682

2. Ohno S: Evolution by Gene Duplication Springer-Verlag; 1970.

3. Hughes MK, Hughes AL: Evolution of duplicate genes in a tetraploid animal, Xenopus laevis. Mol Biol Evol 1993, 10:1360-1369.

4. Cronn RC, Small RL, Wendel JF: Duplicated genes evolve independently after polyploid formation in cotton. Proc Natl Acad Sci USA 1999, 96:14406-14411.

5. Robinson-Rechavi M, Laudet V: Evolutionary rate of duplicate genes in fish and mammals. Mol Biol Evol 2001, 18:681-683.

6. Kondrashov FA, Rogozin IB, Wolf YI, Koonin EV: Selection in the evolution of gene duplications. Genome Biol 2002, 3:RESEARCH0008.

7. Zhang L, Vision TJ, Gaut BS: Patterns of nucleotide substitution among simultaneously duplicated gene pairs in Arabidopsis thaliana. Mol Biol Evol 2002, 19:1464-1473.
8. Lynch M, Katju V: The altered evolutionary trajectories of gene duplicates. Trends Genet 2004, 20:544-549.

9. Van de Peer $Y$, Taylor J, Braasch I, Meyer A: The ghost of selection past: rates of evolution and functional divergence of anciently duplicated genes. J Mol Evol 2001, 53:436-446.

10. Nembaware V, Crum K, Kelso J, Seoighe C: Impact of the presence of paralogs on sequence divergence in a set of mouse-human orthologs. Genome Res 2002, 12:1370-1376.

11. Conant GC, Wagner A: Asymmetric sequence divergence of duplicate genes. Genome Res 2003, 13:2052-2058.

12. Kellis M, Birren BW, Lander ES: Proof and evolutionary analysis of ancient genome duplication in the yeast Saccharomyces cerevisae. Nature 2004, 428:617-624.

13. Kim S-O, Yi SV: Correlated asymmetry of sequence and functional divergence between duplicate proteins of Saccharomyces cerevisiae. Mol Biol Evol 2006, 23:1068-1075.

14. Katju V, Lynch M: The structure and early evolution of recently arisen gene duplicates in the Caenorhabditis elegans genome. Genetics 2003, 165:1793-1803.

15. Katju V, Lynch M: On the formation of novel genes by duplication in the Caenorhabditis elegans genome. Mol Biol Evol 2006, 23:1056-1067.

16. Cusack BP, Wolfe KH: Not born equal: increased asymmetry in relocated and retrotransposed rodent gene duplicates. Mol Biol Evol 2007, 24:679-686.

17. Han MV, Demuth JP, McGrath CL, Casola C, Hahn MW: Adaptive evolution of young gene duplicates in mammals. Genome Res 2009, 19:859-867.

18. Wolfe KH, Shields DC: Molecular evidence for an ancient duplication of the entire yeast genome. Nature 1997, 387:708-713.

19. Sharp PM, Li WH: The Codon Adaptation Index - a measure of directional synonymous codon usage bias and its potential applications. NuCl Acids Res 1987, 11:1281-1295.

20. Kondrashov FA, Rogozin IB, Wolf YI, Koonin EV: Selection in the evolution of gene duplications. Genome Biol 2002, 3:0008.1.

21. Wagner A: Asymmetric functional divergence of duplicate genes in yeast. Mol Biol Evol 2002, 19:1760-1768.

22. Zhang $\mathrm{P}, \mathrm{Gu} Z \mathrm{Z}, \mathrm{Li}$ W-H: Different evolutionary patterns between young gene duplicates in the human genome. Genome Biol 2003, 4:R56.

23. Scannell DR, Wolfe $\mathrm{KH}$ : A burst of protein sequence evolution and a prolonged period of asymmetric evolution follow gene duplication in yeast. Genome Res 2008, 18:137-147.

24. Panchin AY, Gelfand MS, Ramensky VE, Artamanova IE: Asymmetric and non-uniform evolution of recently duplicated human genes. Biol Dir 2010, 5:54

25. Notebaart RA, Huynen MA, Teusink B, Siezen RJ, Snel B: Correlation between sequence conservation and the genomic context after gene duplication. Nucl Acids Res 2005, 33:6164-6171

26. Cliften P, Fulton RS, Wilson RK, Johnston M: After the duplication: gene loss and adaptation in Saccharomyces genomes. Genetics 2006, 172:863-872.

27. Scannell DR, Byrne KP, Gordon JL, Wong S, Wolfe KH: Multiple rounds of speciation associated with reciprocal gene loss in polyploid yeasts. Nature 2006, 440:341-345

28. Abi-Rached L, Gilles A, Shiina T, Pontarotti P, Inoko H: Evidence of en bloc duplication in vertebrate genomes. Nat Genetics 2002, 31:100-105.

29. Drummond DA, Bloom JD, Adami C, Wilke CO, Arnold FH: Why highly expressed proteins evolve slowly. Proc Natl Acad Sci USA 2005 102:14338-14343.

30. Drummond DA, Raval A, Wilke CO: A single determinant dominates the rate of yeast protein evolution. Mol Biol Evol 2006, 23:327-337.

31. Katju V, Farslow JC, Bergthorsson U: Variation in gene duplicates with low synonymous divergence in Saccharomyces cerevisiae relative to Caenorhabditis elegans. Genome Biol 2009, 10:R75.

32. Conant GC, Wagner A: GenomeHistory: a software tool and its application to fully sequenced genomes. Nucl Acids Res 2002, 30:3378-3386

33. Wolfe K: Robustness - it's not where you think it is. Nat Genet 2000, 25:3-4.

34. Wong S, Butler G, Wolfe KH: Gene order evolution and paleopolyploidy in hemiascomycete yeasts. Proc Natl Acad Sci USA 2002, 99:9272-9277.

35. Dietrich FS, Voegeli S, Brachat S, Lerch A, Gates K, Steiner S, Mohr C, Pöhlmann R, Luedi P, Choi S, Wing RA, Flavier A, Gaffney TD, Philippsen P: 
The Ashbya gossypii genome as a tool for mapping the ancient Saccharomyces cerevisiae genome. Science 2004, 304:304-307.

36. Byrne KP, Wolfe KH: The Yeast Gene Order Browser: combining curated homology and syntenic context reveals gene fate in polyploid species. Genome Res 2005, 15:1456-1461.

37. Gordon JL, Byrne KP, Wolfe KH: Additions, losses and rearrangements on the evolutionary route from a reconstructed ancestor to the modern Saccharomyces cerevisiae genome. PLoS Genet 2009, 5:e1000485.

38. Yang Z, Kumar S, Nei M: A new method of inference of ancestral nucleotide and amino acid sequences. Genetics 1995, 141:1641-1650.

39. Koshi JM, Goldstein RA: Probabilistic reconstruction of ancestral protein sequences. J Mol Evol 1996, 42:313-320.

40. Yang Z: Computational Molecular Evolution Oxford University Press, Oxford, England; 2006

41. Tajima F: Simple methods for testing the molecular evolutionary clock hypothesis. Genetics 1993, 135:599-607.

42. Tamura K, Dudley J, Nei M, Kumar S: MEGA4: Molecular Evolutionary Genetics Analysis (MEGA) software version 4.0. Mol Biol Evol 2007. 24:1596-1599.

43. Grote A, Hiller K, Scheer M, Münch R, Nörtemann B, Hempel DC, Jahn D: JCat: a novel tool to adapt codon usage of a target gene to its potential expression host. Nucl Acids Res 2005, 33:W526-W531.

44. Carbone A, Zinovyev A, Képès F: Codon adaptation index as a measure of dominating codon bias. Bioinformatics 2003, 19:2005-2015.

45. Lipson D, Raz T, Kieu A, Jones DR, Giladi E, Thayer E, Thompson JF, Letovsky S, Milos P, Causey M: Quantification of the yeast transcriptome by single-molecule sequencing. Nat Biotech 2009, 27:652-658.

doi:10.1186/1471-2148-11-279

Cite this article as: Bu et al:: Local synteny and codon usage contribute to asymmetric sequence divergence of Saccharomyces cerevisiae gene duplicates. BMC Evolutionary Biology 2011 11:279.

\section{Submit your next manuscript to BioMed Central and take full advantage of:}

- Convenient online submission

- Thorough peer review

- No space constraints or color figure charges

- Immediate publication on acceptance

- Inclusion in PubMed, CAS, Scopus and Google Scholar

- Research which is freely available for redistribution

Submit your manuscript at www.biomedcentral.com/submit 